\title{
INVARIANT MEASURES FOR THE LINEAR STOCHASTIC CAUCHY PROBLEM AND $R$-BOUNDEDNESS OF THE RESOLVENT
}

\author{
J.M.A.M. VAN NEERVEN AND L. WEIS
}

Dedicated to Giuseppe Da Prato on the occasion of his 70th birthday

\begin{abstract}
We study the asymptotic behaviour of solutions of the stochastic abstract Cauchy problem

$$
\left\{\begin{aligned}
d U(t) & =A U(t) d t+B d W_{H}(t), \quad t \geqslant 0 \\
U(0) & =0
\end{aligned}\right.
$$

where $A$ is the generator of a $C_{0}$-semigroup on a Banach space $E, W_{H}$ is a cylindrical Brownian motion over a separable Hilbert space $H$, and $B \in$ $\mathscr{L}(H, E)$ is a bounded operator. Assuming the existence of a solution $U$, we prove that a unique invariant measure exists if the resolvent $R(\lambda, A)$ is $R$ bounded in the right half-plane $\{\operatorname{Re} \lambda>0\}$, and that conversely the existence of an invariant measure implies the $R$-boundedness of $R(\lambda, A) B$ in every halfplane properly contained in $\{\operatorname{Re} \lambda>0\}$. We study various abscissae related to the above problem and show, among other things, that the abscissa of $R$ boundedness of the resolvent of $A$ coincides with the abscissa corresponding to the existence of invariant measures for all $\gamma$-radonifying operators $B$ provided the latter abscissa is finite. For Hilbert spaces $E$ this result reduces to the Gearhart-Herbst-Prüss theorem.
\end{abstract}

\section{INTRODUCTION AND STATEMENT OF THE RESULTS}

Let $A$ be the generator of a $C_{0}$-semigroup $S=\{S(t)\}_{t \geqslant 0}$ on a Banach space $E$. Denoting the abscissa of uniform boundedness of the resolvent by $s_{0}(A)$ and the growth bound by $\omega_{0}(A)$, cf. [2, 22], the easy part of the Hille-Yosida theorem implies that $s_{0}(A) \leqslant \omega_{0}(A)$. A classical theorem of Gearhart, Herbst, and Prüss $[12,16,28]$ states that in Hilbert spaces $E$, equality $s_{0}(A)=\omega_{0}(A)$ holds. More precisely, if the resolvent $R(\lambda, A)=(\lambda-A)^{-1}$ is uniformly bounded on $\{\operatorname{Re} \lambda>0\}$, then $S$ is uniformly exponentially stable. The main result of this paper is a version of the Gearhart-Herbst-Prüss theorem for the linear stochastic Cauchy problem

$$
\left\{\begin{aligned}
d U(t) & =A U(t) d t+B d W_{H}(t), \quad t \geqslant 0, \\
U(0) & =0
\end{aligned}\right.
$$

2000 Mathematics Subject Classification. Primary: 47D03; Secondary: 35R15, 47D06, $60 \mathrm{H} 05$.

Key words and phrases. $R$-bounded resolvent, invariant measures, $C_{0}$-semigroups, stochastic evolution equations in Banach spaces.

The first named author gratefully acknowledges support by a 'VIDI subsidie' in the 'Vernieuwingsimpuls' programme of the Netherlands Organization for Scientific Research (NWO) and by the Research Training Network HPRN-CT-2002-00281. The second named author was supported by grants from the Volkswagenstiftung (I/78593) and the Deutsche Forschungsgemeinschaft (We 2847/1-1). 
where $W_{H}$ is a cylindrical Brownian motion over a separable real Hilbert space $H$ and $B \in \mathscr{L}(H, E)$ is a fixed operator. The notion of a cylindrical Brownian motion, as well as other unexplained notions used in this introduction, will be explained in later sections.

Theorem 1.1. Assume that the problem $\left(\mathrm{SCP}_{B}\right)$ has a solution. If the resolvent $R(\lambda, A)$ is $\gamma$-bounded on $\{\operatorname{Re} \lambda>0\}$, then $\left(\mathrm{SCP}_{B}\right)$ admits a unique invariant measure.

In particular an invariant measure exists under the stronger assumption that the resolvent $R(\lambda, A)$ is $R$-bounded on $\{\operatorname{Re} \lambda>0\}$.

The existence of an invariant measure implies that the solution $U$ is bounded in all means. This will be elaborated further in Section 4 .

In the converse direction we prove:

Theorem 1.2. If the problem $\left(\mathrm{SCP}_{B}\right)$ admits an invariant measure, then $R(\lambda, A) B$ has an analytic extension to $\{\operatorname{Re} \lambda>0\}$ which is $R$-bounded on $\{\operatorname{Re} \lambda \geqslant \delta\}$ for every $\delta>0$, with an $R$-bound of order $O(1 / \sqrt{\delta})$ as $\delta \downarrow 0$.

In some sense Theorems 1.1 and 1.2 are optimal even if $E$ is a Hilbert space, as is shown by the following example [15, Example 7.1].

Example 1. Let $H=E=\ell^{2}$ with standard unit basis $\left(u_{n}\right)_{n \geqslant 1}$. Let $\left(b_{n}\right)_{n \geqslant 1}$ be a bounded sequence of positive real numbers and define $B \in \mathscr{L}(H, E)$ by $B u_{n}:=$ $b_{n} u_{n}$. Let $\left(a_{n}\right)_{n \geqslant 1}$ be a sequence of positive real numbers and define the operator $A$ with maximal domain $\mathscr{D}(A)$ by $A u_{n}:=-a_{n} u_{n}$. Then $A$ generates a $C_{0}$-semigroup $S$ on $E$ given by $S(t) u_{n}=e^{-a_{n} t} u_{n}$.

- Take $b_{n}=1 / n$ and $a_{n}=1 / \sqrt{n}$. Then the problem $\left(\mathrm{SCP}_{B}\right)$ admits a solution, for all $\delta>0$ the resolvent $R(\lambda, A)$ is $(R$-)bounded on $\{\operatorname{Re} \lambda \geqslant \delta\}$, but no invariant measure exists.

- Take $b_{n}=1 / n \sqrt{n}$ and $a_{n}=1 / \sqrt{n}$. Then the problem $\left(\mathrm{SCP}_{B}\right)$ admits a unique invariant measure, but $R(\lambda, A) B$ is $(R$-)unbounded on $\{\operatorname{Re} \lambda>0\}$.

Remark 2. A solution of $\left(\mathrm{SCP}_{B}\right)$ always exists under the following assumptions:

- $B$ is $\gamma$-radonifying and $A$ generates an analytic $C_{0}$-semigroup [10];

- $B$ is $\gamma$-radonifying and $E$ has type 2 [25];

- $B$ is $\gamma$-radonifying, $E$ has property $\left(\alpha^{+}\right)$, and $\left(\mathrm{SCP}_{C}\right)$ admits a solution for all rank 1 operators $C: H \rightarrow E[26]$.

For $\gamma$-radonifying operators $B$ the problem $\left(\mathrm{SCP}_{B}\right)$ may be equivalently reformulated as

$\left(\mathrm{SCP}_{W}\right) \quad\left\{\begin{aligned} d U(t) & =A U(t) d t+d W(t), \quad t \geqslant 0, \\ U(0) & =0,\end{aligned}\right.$

where $W$ is the unique $E$-valued Brownian motion satisfying

$$
\left\langle W(t), x^{*}\right\rangle=W_{H}(t) B^{*} x^{*}, \quad t \geqslant 0, x^{*} \in E^{*} .
$$

Conversely every problem of the form $\left(\mathrm{SCP}_{W}\right)$, with $W$ an $E$-valued Brownian motion, may be reformulated in the form $\left(\mathrm{SCP}_{B}\right)$, where $B: H \hookrightarrow E$ is the $\gamma$ radonifying embedding of the reproducing kernel Hilbert space $H$ associated with $B$. We refer to $[24,26]$ for more details. 
If a solution of $\left(\mathrm{SCP}_{B}\right)$ exists, it is unique up to modification. Even if $B$ is a rank 1 operator, solutions may fail to exist, however; examples are presented in $[9,24]$ and in Example 8 below.

Theorem 1.3. Assume that the problem $\left(\mathrm{SCP}_{B}\right)$ admits an invariant measure for all rank 1 operators $B \in \mathscr{L}(H, E)$. Then $\{\operatorname{Re} \lambda>0\} \subseteq \varrho(A)$ and the resolvent $R(\lambda, A)$ is $R$-bounded on $\{\operatorname{Re} \lambda \geqslant \delta\}$ for every $\delta>0$, with an $R$-bound of order $O(1 / \sqrt{\delta})$ as $\delta \downarrow 0$.

If $\left(\mathrm{SCP}_{B}\right)$ admits an invariant measure for all $\gamma$-radonifying operators $B \in$ $\mathscr{L}(H, E)$ a stronger conclusion holds; see Remark 10 at the end of the paper.

Theorems 1.2 and 1.3 are deduced from an abstract result on the $R$-boundedness of operator-valued Laplace transforms, presented in Section 3. The notion of $R$ boundedness has been studied recently by many authors and has played a crucial role in the solution of the maximal regularity problem for parabolic evolution equations in Banach spaces; cf. $[5,8,19,32]$ and the references given therein. Every $R$-bounded family of operators is $\gamma$-bounded and every $\gamma$-bounded family is uniformly bounded.

Motivated by the above results we introduce the abscissae

$$
\begin{aligned}
& s_{\gamma}^{B}(A):=\inf \{\omega>s(A): \lambda \mapsto R(\lambda, A) B \text { has a } \gamma \text {-bounded } \\
&\text { analytic extension to }\{\operatorname{Re} \lambda>\omega\}\}, \\
& s_{R}^{B}(A):=\inf \{\omega>s(A): \lambda \mapsto R(\lambda, A) B \text { has an } R \text {-bounded } \\
&\text { analytic extension to }\{\operatorname{Re} \lambda>\omega\}\},
\end{aligned}
$$

where $B \in \mathscr{L}(H, E)$ is fixed, and

$$
\begin{aligned}
& s_{\gamma}(A):=\inf \{\omega>s(A): \lambda \mapsto R(\lambda, A) \text { is } \gamma \text {-bounded on }\{\operatorname{Re} \lambda>\omega\}\}, \\
& s_{R}(A):=\inf \{\omega>s(A): \lambda \mapsto R(\lambda, A) \text { is } R \text {-bounded on }\{\operatorname{Re} \lambda>\omega\}\} .
\end{aligned}
$$

We use the convention that the infimum over the empty set equals $\infty$. Clearly,

$$
s_{\gamma}^{B}(A) \leqslant s_{R}^{B}(A) \text { and } s_{0}(A) \leqslant s_{\gamma}(A) \leqslant s_{R}(A) .
$$

An example showing that strict inequality $s_{0}(A)<s_{\gamma}(A)$ may occur is given in [17]. No example seems to be known of a generator $A$ for which $s_{\gamma}(A)<s_{R}(A)$ holds. If $E$ has finite cotype, then Gaussian sums and Rademacher sums are comparable and therefore equality $s_{\gamma}(A)=s_{R}(A)$ holds. It will follow from Theorem 1.5 that $s_{\gamma}(A)=s_{R}(A)$ also holds if $\left(\mathrm{SCP}_{B}\right)$ has a solution for all rank 1 operators $B$.

Example 3. If $A$ is the generator of a positive $C_{0}$-semigroup on a Banach lattice $E$ which is $q$-concave with $1 \leqslant q<\infty$, then $s(A)=s_{0}(A)=s_{\gamma}(A)=s_{R}(A)[14$, Example 5.5(b)].

As an application of Theorem 1.1 we shall construct next an example of a $C_{0}$-semigroup with positive growth bound which has the property that for all $\gamma$ radonifying operators $B$, the problem $\left(\mathrm{SCP}_{B}\right)$ has an invariant measure. This remarkable phenomenon cannot occur in Hilbert spaces, and more generally in cotype 2 spaces; cf. Example 7 below. 
Example 4. For $1 \leqslant p \leqslant q<\infty$ consider the space $E:=L^{p}(1, \infty) \cap L^{q}(1, \infty)$ endowed with the norm $\|f\|:=\max \left\{\|f\|_{p},\|f\|_{q}\right\}$. On $E$ we define the $C_{0}$-semigroup $S$ by

$$
(S(t) f)(s):=f\left(s e^{t}\right), \quad s>1, t \geqslant 0 .
$$

It was shown by Arendt [1] that

$$
s_{0}(A)=-\frac{1}{p}<-\frac{1}{q}=\omega_{0}(A) .
$$

By Example 3, $s_{\gamma}(A)=s_{R}(A)=-\frac{1}{p}$. Now let $2 \leqslant p<q<\infty$ and put $S_{c}(t):=$ $e^{c t} S(t)$ and $A_{-c}:=A+c$, where $\frac{1}{q}<c<\frac{1}{p}$ is an arbitrary but fixed number. Then $E$ has type 2 and the problem $\left(\mathrm{SCP}_{B}\right)$ with $A$ replaced by $A_{-c}$ has a solution for all $\gamma$-radonifying operators $B$, cf. Remark 2. In view of $s_{\gamma}\left(A_{-c}\right)=-\frac{1}{p}+c<0$, Theorem 1.1 shows that an invariant measure always exists. On the other hand, $\omega_{0}\left(A_{-c}\right)=-\frac{1}{q}+c>0$.

For a fixed operator $B \in \mathscr{L}(H, E)$ we introduce the following abscissa for the existence of an invariant measure for the problem $\left(\mathrm{SCP}_{B}\right)$ :

$$
\begin{aligned}
\omega_{\text {inv }}^{B}(A):=\inf \{\omega \in \mathbb{R}: & \text { the problem }\left(\mathrm{SCP}_{B}\right) \text { with } A \text { replaced } \\
& \text { by } A-\omega \text { admits an invariant measure }\} .
\end{aligned}
$$

In Section 4 it will be shown that $\omega_{\text {inv }}^{B}(A)<\infty$ if and only if $\left(\mathrm{SCP}_{B}\right)$ has a solution, in which case $\omega_{\text {inv }}^{B}(A)$ is equal to the abscissa of existence of a solution of $\left(\mathrm{SCP}_{B}\right)$ which is bounded in $p$-th moment for some (all) $p \in[1, \infty)$. In terms of the abscissa $\omega_{\text {inv }}^{B}(A)$, the main assertions of Theorems 1.1 and 1.2 admit the following functional analytic formulation.

Theorem 1.4. If the problem $\left(\mathrm{SCP}_{B}\right)$ admits a solution, then

$$
s_{\gamma}^{B}(A) \leqslant s_{R}^{B}(A) \leqslant \omega_{\text {inv }}^{B}(A) \leqslant s_{\gamma}(A) \leqslant s_{R}(A) .
$$

In view of Remark 2 it is natural to define two more abscissae related to the existence of invariant measures, viz.

$$
\begin{aligned}
\omega_{\text {inv }}^{(1)}(A):=\inf \{\omega \in \mathbb{R}: & \text { the problem }\left(\mathrm{SCP}_{B}\right) \text { with } A \text { replaced } \\
& \text { by } A-\omega \text { admits an invariant measure } \\
& \text { for all rank } 1 \text { operators } B \in \mathscr{L}(H, E)\}, \\
\omega_{\text {inv }}^{\gamma}(A):=\inf \{\omega \in \mathbb{R}: & \text { the problem }\left(\mathrm{SCP}_{B}\right) \text { with } A \text { replaced } \\
& \text { by } A-\omega \text { admits an invariant measure } \\
& \text { for all } \gamma \text {-radonifying operators } B \in \mathscr{L}(H, E)\} .
\end{aligned}
$$

We have $\omega_{\text {inv }}^{(1)}(A)<\infty$ (resp. $\left.\omega_{\text {inv }}^{\gamma}(A)<\infty\right)$ if and only if $\left(\mathrm{SCP}_{B}\right)$ has a solution for all rank 1 (resp. $\gamma$-radonifying) operators $B$.

\section{Theorem 1.5.}

(1) If the problem $\left(\mathrm{SCP}_{B}\right)$ admits a solution for all rank 1 operators $B \in$ $\mathscr{L}(H, E)$, then

$$
s_{0}(A) \leqslant s_{\gamma}(A)=s_{R}(A)=\omega_{\text {inv }}^{(1)}(A) \leqslant \omega_{0}(A) .
$$


(2) If the problem $\left(\mathrm{SCP}_{B}\right)$ admits a solution for all $\gamma$-radonifying operators $B \in \mathscr{L}(H, E)$, then

$$
s_{0}(A) \leqslant s_{\gamma}(A)=s_{R}(A)=\omega_{\text {inv }}^{(1)}(A)=\omega_{\text {inv }}^{\gamma}(A) \leqslant \omega_{0}(A) .
$$

Example 5. If $E$ is a Hilbert space, then Theorem 1.5 reduces to the GearhartHerbst-Prüss theorem. To see this, first note that on the one hand we have

$$
s_{0}(A)=s_{\gamma}(A)=s_{R}(A)
$$

since the notions of uniform boundedness, $\gamma$-boundedness, and $R$-boundedness agree for Hilbert spaces. On the other hand, $\left(\mathrm{SCP}_{B}\right)$ has a solution for all $\gamma$ radonifying operators $B$. If $B$ is a rank 1 operator, say $B h=\left[h, h_{0}\right]_{H} x_{0}$ for $h \in H$, then by Proposition 4.4 below an invariant measure for $\left(\mathrm{SCP}_{B}\right)$ exists with $A$ replaced by $A-\omega$ if and only if the orbit $t \mapsto e^{-\omega t} S(t) x_{0}$ belongs to $L^{2}\left(\mathbb{R}_{+} ; E\right)$. The Datko-Pazy theorem therefore implies that

$$
\omega_{\text {inv }}^{(1)}(A)=\omega_{\text {inv }}^{\gamma}(A)=\omega_{0}(A)
$$

Example 6. If $A$ is the generator of a $C_{0}$-semigroup on a real Banach space $E$ and $\left(\mathrm{SCP}_{B}\right)$ has a solution for all rank 1 (resp. $\gamma$-radonifying) operators $B$, then $s(A)=s_{0}(A)=s_{R}(A)=s_{\gamma}(A)=\omega_{\text {inv }}^{(1)}(A)\left(=\omega_{\text {inv }}^{\gamma}(A)\right)=\omega_{0}(A)$ under each of the following additional assumptions:

- $S$ is eventually norm continuous;

- $S$ is positive on $E=C_{0}(\Omega)$ with $\Omega$ locally compact Hausdorff;

- $S$ is positive on $E=L^{p}$ with $p \in[1, \infty)$.

Indeed, well-known results from semigroup theory imply that in each of these cases we have $s(A)=\omega_{0}(A)$ and the result follows from Theorem 1.5.

It follows from Example 4 that under the assumption of Theorem 1.5, strict inequality $\omega_{\text {inv }}^{\gamma}(A)<\omega_{0}(A)$ may occur. On the other hand, the next example shows that in cotype 2 spaces one always has $\omega_{\text {inv }}^{(1)}(A)=\omega_{0}(A)$ provided the former abscissa is finite.

Example 7. If $E$ has cotype 2 and $\omega_{\text {inv }}^{(1)}(A)<\infty$, then $s_{R}(A)=s_{\gamma}(A)=\omega_{\text {inv }}^{(1)}(A)=$ $\omega_{0}(A)$. To see this, let $\omega_{\text {inv }}^{(1)}(A)<c$. It will be enough to prove that $\omega_{0}(A)<c$. Fix $x_{0} \in E$ arbitrary and consider the rank 1 operator $B h=\left[h, h_{0}\right]_{H} x_{0}$. By Proposition 4.4 , the function $t \mapsto e^{-c t} S(t) x_{0}$ belongs to the space $\gamma\left(\mathbb{R}_{+} ; E\right)$, which is introduced in Section 2. Since $E$ has cotype 2, by a result of Rosiński and Suchanecki [29] this implies that $t \mapsto e^{-c t} S(t) x_{0}$ belongs to $L^{2}\left(\mathbb{R}_{+} ; E\right)$; cf. also [24]. Since $x_{0} \in E$ is arbitrary, the Datko-Pazy theorem now shows that $\omega_{0}(A)<c$.

We show next how Examples 3 and 7 may be combined to derive nonexistence results for the problem $\left(\mathrm{SCP}_{B}\right)$.

Example 8. Let $1 \leqslant p<2$ and consider the generator $A$ in $L^{p}(1, \infty)$ of the semigroup $S$ defined by

$$
(S(t) f)(s):=f\left(s e^{t}\right), \quad s>1, t \geqslant 0 .
$$

We take $H=\mathbb{R}$. For $g \in L^{p}(1, \infty)$ let $B_{g} \in \mathscr{L}\left(\mathbb{R}, L^{p}(1, \infty)\right)$ be given by $B_{g} 1:=g$. We shall prove that there exists a function $g \in L^{p}(1, \infty) \cap L^{2}(1, \infty)$ such that the problem $\left(\mathrm{SCP}_{B_{g}}\right)$ fails to have a solution in $L^{p}(1, \infty)$.

To this end let $E:=L^{p}(1, \infty) \cap L^{2}(1, \infty)$. We claim that in $E$, the problem $\left(\mathrm{SCP}_{B_{g_{0}}}\right)$ fails to have a solution for some $g_{0} \in E$. Indeed, otherwise we would have 
$s\left(A_{E}\right)=\omega_{\text {inv }}^{(1)}\left(A_{E}\right)$ by Example 3 and Theorem 1.5, where $A_{E}$ denotes the part of $A$ in $E$. But since $E$ has cotype 2, by Example 7 we have $\omega_{\text {inv }}^{(1)}\left(A_{E}\right)=\omega_{0}\left(A_{E}\right)$. It would follow with (1.1) that $-\frac{1}{p}=s\left(A_{E}\right)=\omega_{\text {inv }}^{(1)}\left(A_{E}\right)=\omega_{0}\left(A_{E}\right)=-\frac{1}{2}$, a contradiction. This proves the claim.

In $L^{2}(1, \infty)$, the problem $\left(\mathrm{SCP}_{B_{g_{0}}}\right)$ does have a solution, cf. Remark 2 . It follows that $\left(\mathrm{SCP}_{B_{g_{0}}}\right)$ fails to have a solution in $L^{p}(1, \infty)$. For otherwise Proposition 4.1 would guarantee the existence of a solution in $L^{p}(1, \infty) \cap L^{2}(1, \infty)=E$, which contradicts the choice of $g_{0}$.

Together with Example 3, this example also shows that $s_{R}(A)<\infty$ may occur even if $\omega_{\text {inv }}^{(1)}(A)=\infty$. In particular, the finiteness of the abscissa $s_{R}(A)$ gives no guarantee for the existence of solutions of $\left(\mathrm{SCP}_{B}\right)$.

Acknowledgment - The first named author thanks Zdzisław Brzeźniak for his kind invitation to present the results of this paper at the East Midlands Stochastic Analysis Seminar (June, 2004). Both authors thank Mark Veraar for some helpful comments.

\section{2. $\gamma$-RADONIFYING OPERATORS}

Solutions of $\left(\mathrm{SCP}_{B}\right)$, if they exist, are Gaussian processes. This explains the important role played by the operator ideal of $\gamma$-radonifying operators in the study of $\left(\mathrm{SCP}_{B}\right)$. In this section we review some of its properties which shall be used throughout this paper. For proofs and more information we refer to [3].

Let $H$ be a separable real Hilbert space and $E$ a real Banach space. A bounded operator $R \in \mathscr{L}(H, E)$ is said to be $\gamma$-radonifying if $R \circ R^{*} \in \mathscr{L}\left(E^{*}, E\right)$ is a Gaussian covariance operator, i.e., if there exists a centred Gaussian Radon measure $\mu$ on $E$ such that

$$
\left\langle R R^{*} x^{*}, y^{*}\right\rangle=\int_{E}\left\langle x, x^{*}\right\rangle\left\langle x, y^{*}\right\rangle d \mu(x) \quad \forall x^{*}, y^{*} \in E^{*} .
$$

If $\left(g_{n}\right)_{n \geqslant 1}$ is a sequence of independent standard normal random variables (briefly, an orthogaussian sequence) on some probability space $(\Omega, \mathscr{F}, \mathbb{P})$ and $\left(h_{n}\right)_{n \geqslant 1}$ is an orthonormal basis of $H$, then $R \in \mathscr{L}(H, E)$ is $\gamma$-radonifying if and only if the series $\sum_{n \geqslant 1} g_{n} R h_{n}$ converges in $L^{2}(\Omega ; E)$; the distribution $\mu_{R}$ of its sum is then a centred Gaussian Radon measure on $E$ with covariance $R \circ R^{*}$. The space $\gamma(H, E)$ of all $\gamma$-radonifying operators from $H$ into $E$ is a Banach space with respect to the norm $\|\cdot\|_{\gamma(H, E)}$ defined by

$$
\|R\|_{\gamma(H, E)}^{2}:=\mathbb{E}\left\|\sum_{n \geqslant 1} g_{n} R h_{n}\right\|^{2}=\int_{E}\|x\|^{2} d \mu_{R}(x) .
$$

If $E$ is a Hilbert space, then $\gamma(H, E)=\mathscr{L}_{2}(H, E)$ with equal norms.

By Anderson's inequality, any positive symmetric operator which is dominated by a Gaussian covariance is itself a Gaussian covariance. More precisely, let $Q_{1}, Q_{2} \in$ $\mathscr{L}\left(E^{*}, E\right)$ be positive symmetric operators satisfying

$$
\left\langle Q_{1} x^{*}, x^{*}\right\rangle \leqslant\left\langle Q_{2} x^{*}, x^{*}\right\rangle
$$

for all $x^{*} \in E^{*}$. Then $Q_{1}$ is a Gaussian covariance if $Q_{2}$ is a Gaussian covariance. Moreover, if in this situation $R_{1}: H_{1} \rightarrow E$ and $R_{2}: H_{2} \rightarrow E$ satisfy $R_{1} \circ R_{1}^{*}=Q_{1}$ and $R_{2} \circ R_{2}^{*}=Q_{2}$, then $R_{1}$ and $R_{2}$ are $\gamma$-radonifying and

$$
\left\|R_{1}\right\|_{\gamma\left(H_{1}, E\right)} \leqslant\left\|R_{2}\right\|_{\gamma\left(H_{2}, E\right)} .
$$


A simple consequence of Anderson's inequality is the following ideal property of Gaussian covariances: if $S \in \mathscr{L}\left(H_{1}, H\right), R \in \gamma(H, E)$, and $T \in \mathscr{L}\left(E, E_{1}\right)$, then $T \circ R \circ S \in \gamma\left(H_{1}, E_{1}\right)$ and

$$
\|T \circ R \circ S\|_{\gamma\left(H_{1}, E_{1}\right)} \leqslant\|T\|\|R\|_{\gamma(H, E)}\|S\| .
$$

In particular every bounded operator $S: H_{1} \rightarrow H_{2}$ induces a bounded operator $\widetilde{S}: \gamma\left(H_{1}, E\right) \rightarrow \gamma\left(H_{2}, E\right)$ by the formula

$$
\widetilde{S} R:=R \circ S^{*} .
$$

Moreover,

$$
\|\widetilde{S}\|_{\mathscr{L}\left(\gamma\left(H_{1}, E\right), \gamma\left(H_{2}, E\right)\right)} \leqslant\|S\|_{\mathscr{L}\left(H_{1}, H_{2}\right)} .
$$

This extension procedure has been introduced in [18] and will be applied below to the Fourier-Plancherel transform.

Let $(M, m)$ be a separable and $\sigma$-finite measure space. We say that a function $\phi: M \rightarrow E$ is weakly $L^{2}$ if $\left\langle\phi, x^{*}\right\rangle \in L^{2}(M)$ for all $x^{*} \in E^{*}$. Such a function is said to represent an operator $R \in \mathscr{L}\left(L^{2}(M), E\right)$ if for all $f \in L^{2}(M)$ and $x^{*} \in E^{*}$ we have

$$
\left\langle R f, x^{*}\right\rangle=\int_{M} f(t)\left\langle\phi(t), x^{*}\right\rangle d m(t) .
$$

Following [18], the vector space of all weakly $L^{2}$-functions $\phi$ representing an element $R$ of $\gamma\left(L^{2}(M), E\right)$ is denoted by $\gamma(M ; E)$. We identify functions representing the same operator. Endowed with the norm

$$
\|\phi\|_{\gamma(M ; E)}:=\|R\|_{\gamma\left(L^{2}(M), E\right)},
$$

$\gamma(M ; E)$ is isometric with a dense subspace of $\gamma\left(L^{2}(M), E\right)$. We will frequently apply Anderson's inequality in the following form: if $\phi: M \rightarrow E$ and $\psi: M \rightarrow E$ are weakly $L^{2}$ and satisfy

$$
\int_{M}\left\langle\phi(t), x^{*}\right\rangle^{2} d m(t) \leqslant \int_{M}\left\langle\psi(t), x^{*}\right\rangle^{2} d m(t) \quad \forall x^{*} \in E^{*},
$$

then $\psi \in \gamma(M ; E)$ implies $\phi \in \gamma(M ; E)$ and we have $\|\phi\|_{\gamma(M ; E)} \leqslant\|\psi\|_{\gamma(M ; E)}$. As a special case we have the following ideal property for $\gamma(M ; E)$ : if $a \in L^{\infty}(M)$ and $\phi \in \gamma(M ; E)$, then $a \phi \in \gamma(M ; E)$ and

$$
\|a \phi\|_{\gamma(M ; E)} \leqslant\|a\|_{\infty}\|\phi\|_{\gamma(M ; E)} .
$$

We say that a function $\phi: M \rightarrow \mathscr{L}(H, E)$ is $H$-weakly $L^{2}$ if $\phi^{*} x^{*} \in L^{2}(M ; H)$ for all $x^{*} \in E^{*}$; such a function is said to represent an operator $R \in \mathscr{L}\left(L^{2}(M ; H), E\right)$ if for all $f \in L^{2}(M ; H)$ and $x^{*} \in E^{*}$ we have

$$
\left\langle R f, x^{*}\right\rangle=\int_{M}\left[\phi^{*}(t) x^{*}, f(t)\right]_{H} d m(t) .
$$

Again we identify functions representing the same operator. Endowed with the norm

$$
\|\phi\|_{\gamma(M ; H, E)}:=\|R\|_{\gamma\left(L^{2}(M ; H), E\right)},
$$

$\gamma(M ; H, E)$ is isometric with a dense subspace of $\gamma\left(L^{2}(M ; H), E\right)$. 


\section{3. $R$-BOUNDEDNESS AND $\gamma$-BOUNDEDNESS}

Let $\left(r_{n}\right)_{n \geqslant 1}$ be a sequence of independent Rademacher variables on some probability space $(\Omega, \mathscr{F}, \mathbb{P})$. A family of operators $\mathscr{T} \subseteq \mathscr{L}(E)$ is called $R$-bounded if there exists a constant $C$ such that for all $N \geqslant 1$ and all sequences $\left(T_{n}\right)_{n=1}^{N} \subseteq \mathscr{T}$ and $\left(x_{n}\right)_{n=1}^{N} \subseteq E$ we have

$$
\mathbb{E}\left\|\sum_{n=1}^{N} r_{n} T_{n} x_{n}\right\|^{2} \leqslant C^{2} \mathbb{E}\left\|\sum_{n=1}^{N} r_{n} x_{n}\right\|^{2} .
$$

The least possible constant $C$ is called the $R$-bound of $\mathscr{T}$, notation $R(\mathscr{T})$. By replacing the Rademacher sequence $\left(r_{n}\right)_{n \geqslant 1}$ by an orthogaussian sequence $\left(g_{n}\right)_{n \geqslant 1}$ we obtain the corresponding notion of a $\gamma$-bounded family. Its $\gamma$-bound is denoted by $\gamma(\mathscr{T})$.

Every $\gamma$-bounded family $\mathscr{T}$ is uniformly bounded and for all $T \in \mathscr{T}$ we have $\|T\| \leqslant \gamma(\mathscr{T})$. Every $R$-bounded family is $\gamma$-bounded, with $\gamma(\mathscr{T}) \leqslant R(\mathscr{T})$. Indeed, by randomizing with an independent Rademacher sequence $\left(\tilde{r}_{n}\right)_{n \geqslant 1}$ and using Fubini's theorem,

$$
\begin{aligned}
\mathbb{E}\left\|\sum_{n=1}^{N} g_{n} T_{n} x_{n}\right\|^{2}=\tilde{\mathbb{E}} \mathbb{E}\left\|\sum_{n=1}^{N} \tilde{r}_{n} g_{n} T_{n} x_{n}\right\|^{2} \\
=\mathbb{E} \tilde{\mathbb{E}}\left\|\sum_{n=1}^{N} \tilde{r}_{n} g_{n} T_{n} x_{n}\right\|^{2} \leqslant(R(\mathscr{T}))^{2} \mathbb{E} \tilde{\mathbb{E}}\left\|\sum_{n=1}^{N} \tilde{r}_{n} g_{n} x_{n}\right\|^{2} \\
=(R(\mathscr{T}))^{2} \tilde{\mathbb{E}} \mathbb{E}\left\|\sum_{n=1}^{N} \tilde{r}_{n} g_{n} x_{n}\right\|^{2}=(R(\mathscr{T}))^{2} \mathbb{E}\left\|\sum_{n=1}^{N} g_{n} x_{n}\right\|^{2} .
\end{aligned}
$$

In spaces with finite cotype, Rademacher sums and Gaussian sums are comparable [11, Chapter 12] and the notions of $R$-boundedness and $\gamma$-boundedness are equivalent. In Hilbert spaces, both notions are equivalent to uniform boundedness.

If $\mathscr{S}$ and $\mathscr{T}$ are $R$-bounded ( $\gamma$-bounded), then $\mathscr{S} \mathscr{T}=\{S T: S \in \mathscr{S}, T \in \mathscr{T}\}$ is $R$-bounded ( $\gamma$-bounded), and we have

$$
R(\mathscr{S} \mathscr{T}) \leqslant R(\mathscr{S}) R(\mathscr{T}) \quad(\gamma(\mathscr{S} \mathscr{T}) \leqslant \gamma(\mathscr{S}) \gamma(\mathscr{T}))
$$

Moreover, if $\mathscr{T}$ is $R$-bounded ( $\gamma$-bounded), then its closure in the strong operator topology, $\overline{\mathscr{T}}$, is $R$-bounded ( $\gamma$-bounded), and

$$
R(\overline{\mathscr{T}})=R(\mathscr{T}) \quad(\gamma(\overline{\mathscr{T}})=\gamma(\mathscr{T}))
$$

By viewing a complex Banach space as a real Banach space of twice the dimension, the definitions of $R$-boundedness and $\gamma$-boundedness trivially extend to complex Banach spaces. This will be used tacitly at various places where we discuss $R$-boundedness and $\gamma$-boundedness of certain operator-valued analytic functions.

There exist intimate connections between $\gamma$-bounded families and $\gamma$-radonifying operators. As a first illustration of this principle we state a simple extension of a multiplier result from [18].

Proposition 3.1. Let $\mu$ be a $\sigma$-finite Radon measure on a separable metric space $X$. Let $E$ and $F$ be real Banach spaces, and let $N: X \rightarrow \mathscr{L}(E, F)$ a strongly measurable function. Assume that $N$ has $\gamma$-bounded range, with $\gamma$-bound $\gamma(N)$. 
Then for all $\phi \in \gamma(X ; H, E)$ we have $N \phi \in \gamma(X ; H, F)$ and

$$
\|N \phi\|_{\gamma(X ; H, F)} \leqslant \gamma(N)\|\phi\|_{\gamma(X ; H, E)} .
$$

Here, $(N \phi)(\xi):=N(\xi) \phi(\xi)$ for $\xi \in X$.

As a second illustration we shall prove an $R$-boundedness result for the Laplace transform of operators taking values in $\gamma\left(\mathbb{R}_{+} ; E\right)$. We start with two lemmas.

Lemma 3.2. Let $E$ and $F$ be real Banach spaces and let $T_{1}, \ldots, T_{N}$ be operators in $\mathscr{L}(E, F)$. If $C$ is a constant such that

$$
\mathbb{E}\left\|\sum_{n=1}^{N} g_{n} T_{n} x\right\|^{2} \leqslant C^{2}\|x\|^{2} \quad \forall x \in E,
$$

then for all finite sequences $\left(x_{n}\right)_{n=1}^{N}$ in $E$ we have

$$
\mathbb{E}\left\|\sum_{n=1}^{N} r_{n} T_{n} x_{n}\right\|^{2} \leqslant \frac{1}{2} \pi C^{2} \mathbb{E}\left\|\sum_{n=1}^{N} r_{n} x_{n}\right\|^{2} .
$$

Proof. This follows from the estimates

$$
\begin{aligned}
\mathbb{E}\left\|\sum_{n=1}^{N} r_{n} T_{n} x_{n}\right\|^{2} \stackrel{(*)}{\leqslant} \mathbb{E} \tilde{\mathbb{E}}\left\|\sum_{n, m=1}^{N} r_{n} \tilde{r}_{m} T_{m} x_{n}\right\|^{2} \stackrel{(* *)}{\leqslant} \frac{1}{2} \pi \mathbb{E} \tilde{\mathbb{E}}\left\|\sum_{n, m=1}^{N} r_{n} \tilde{g}_{m} T_{m} x_{n}\right\|^{2} \\
=\frac{1}{2} \pi \mathbb{E} \tilde{\mathbb{E}}\left\|\sum_{m=1}^{N} \tilde{g}_{m} T_{m}\left(\sum_{n=1}^{N} r_{n} x_{n}\right)\right\|^{2} \leqslant \frac{1}{2} \pi C^{2} \mathbb{E}\left\|\sum_{n=1}^{N} r_{n} x_{n}\right\|^{2}
\end{aligned}
$$

where in $(*)$ and $(* *)$ we used [13, Lemma 3.12] and [11, Proposition 12.11], respectively.

In the next lemma, $S$ denotes the open strip $\{\lambda \in \mathbb{C}: 0<\operatorname{Re} \lambda<1\}$.

Lemma 3.3. Let $N: \bar{S} \rightarrow \mathscr{L}(E, F)$ be strongly continuous and bounded, and assume that $N$ is harmonic on $S$. If the sets $N_{k}^{\rho}=\{N(k+i(n+\rho)): n \in \mathbb{Z}\}$ are $R$-bounded, uniformly with respect to $k \in\{0,1\}$ and $\rho \in[0,1)$, then for all $0<\eta<1$ the function $N$ is $R$-bounded on the line $\{\operatorname{Re} \lambda=\eta\}$ and there exists a constant $C_{\eta}$, independent of $k$ and $\rho$, such that

$$
R(\{N(\lambda): \operatorname{Re} \lambda=\eta\}) \leqslant C_{\eta} \sup _{\substack{k \in\{0,1\} \\ \rho \in[0,1)}} R\left(N_{k}^{\rho}\right) .
$$

Proof. By the Poisson formula for the strip we have, for $\lambda=\alpha+i \beta$ with $0<\alpha<1$ and $\beta \in \mathbb{R}$,

$$
N(\lambda) x=\sum_{k=0,1} \int_{-\infty}^{\infty} P_{k}(\alpha, \beta-t) N(k+i t) x d t, \quad x \in E,
$$

with

$$
P_{k}(\alpha, s)=\frac{e^{\pi s} \sin (\pi \alpha)}{\sin ^{2}(\pi \alpha)+\left(\cos (\pi \alpha)-(-1)^{k} e^{\pi s}\right)^{2}} .
$$

Fix $0<\eta<1$ arbitrary. For $\lambda_{j} \in S$ with $\operatorname{Re} \lambda_{j}=\eta$ choose $n_{j} \in \mathbb{Z}$ and $\rho_{j} \in[0,1)$ such that $\lambda_{j}=\eta+i\left(n_{j}+\rho_{j}\right)$. For all finite sequences $\left(x_{j}\right)_{j=1}^{N}$ in $E$ we have, using 
the contraction principle for Rademacher sums,

$$
\begin{aligned}
(\mathbb{E} \| & \left.\sum_{j=1}^{N} r_{j} N\left(\lambda_{j}\right) x_{j} \|\right)^{\frac{1}{2}} \\
& =\left\|\sum_{k=0,1} \sum_{j=1}^{N} r_{j} \int_{-\infty}^{\infty} P_{k}\left(\eta, n_{j}+\rho_{j}-t\right) N(k+i t) x_{j} d t\right\|_{L^{2}(\Omega ; E)} \\
& \leqslant \sum_{k=0,1} \int_{-\infty}^{\infty}\left\|\sum_{j=1}^{N} r_{j} P_{k}\left(\eta, \rho_{j}-\tau\right) N\left(k+i\left(n_{j}+\tau\right)\right) x_{j}\right\|_{L^{2}(\Omega ; E)} d \tau \\
& \leqslant \sum_{k=0,1} \int_{-\infty}^{\infty} \sup _{\rho \in[0,1)} P_{k}(\eta, \rho-\tau)\left\|\sum_{j=1}^{N} r_{j} N\left(k+i\left(n_{j}+\tau\right)\right) x_{j}\right\|_{L^{2}(\Omega ; E)} d \tau \\
& \leqslant \sup _{\substack{k \in\{0,1\} \\
\rho \in[0,1)}} R\left(N_{k}^{\rho}\right) \sum_{k=0,1} \int_{-\infty}^{\infty} \sup _{\rho \in[0,1)} P_{k}(\eta, \rho-\tau) d \tau \cdot\left(\mathbb{E}\left\|\sum_{j=1}^{N} r_{j} x_{j}\right\|^{2}\right)^{\frac{1}{2}} .
\end{aligned}
$$

Note that in combination with [32, Proposition 2.8], the stronger result is obtained that $N$ has $R$-bounded range on every strip $\left\{\eta_{1} \leqslant \operatorname{Re} \lambda \leqslant \eta_{2}\right\}$ with $0<\eta_{1} \leqslant$ $\eta_{2}<1$.

For an operator $T \in \mathscr{L}\left(L^{2}\left(\mathbb{R}_{+}\right), E\right)$ we define the Laplace transform $\widehat{T}:\{\operatorname{Re} \lambda>$ $0\} \rightarrow E$ by

$$
\widehat{T}(\lambda):=T e_{\lambda}, \quad \operatorname{Re} \lambda>0,
$$

where $e_{\lambda} \in L^{2}\left(\mathbb{R}_{+}\right)$is the function $e_{\lambda}(t)=e^{-\lambda t}$. It is easily seen that $\widehat{T}$ is weakly analytic, hence analytic, on its domain. For a bounded operator $\Theta: F \rightarrow$ $\mathscr{L}\left(L^{2}\left(\mathbb{R}_{+}\right), E\right)$, where $F$ is another real Banach space, we define the Laplace transform $\widehat{\Theta}:\{\operatorname{Re} \lambda>0\} \rightarrow \mathscr{L}(F, E)$ by

$$
\widehat{\Theta}(\lambda) y:=\widehat{\Theta y}(\lambda), \quad y \in F, \operatorname{Re} \lambda>0 .
$$

Clearly, $\widehat{\Theta}$ is uniformly bounded on every half-plane $\{\operatorname{Re} \lambda \geqslant \delta\}$ with a bound of order $1 / \sqrt{\delta}$ as $\delta \downarrow 0$.

Theorem 3.4. Let $\Theta: F \rightarrow \gamma\left(L^{2}\left(\mathbb{R}_{+}\right), E\right)$ be a bounded operator. Then $\widehat{\Theta}$ is $R$-bounded on every half-plane $\{\operatorname{Re} \lambda \geqslant \delta\}$ and there exists a universal constant $C$ such that

$$
R(\{\widehat{\Theta}(\lambda): \operatorname{Re} \lambda \geqslant \delta\}) \leqslant C\|\Theta\| \max \left\{1, \frac{1}{\sqrt{\delta}}\right\} .
$$

Proof. Let $\delta>0$ and $\min \left\{\frac{1}{4} \delta, \frac{1}{2}\right\} \leqslant r \leqslant \min \left\{\frac{1}{2} \delta, \frac{1}{2}\right\}$ be arbitrary and fixed. For $n \in \mathbb{Z}$ and $\rho \in[0,1)$ let $D_{n}^{\rho}$ denote the disc of radius $r$ with centre $\delta+2 i(n+\rho) r$ and define

$$
f_{n}^{\rho}(s, t):=\frac{1}{\sqrt{\pi r^{2}}} 1_{D_{n}^{\rho}}(s+i t) .
$$

For each $\rho$, the sequence $\left(f_{n}^{\rho}\right)_{n \in \mathbb{Z}}$ is an orthonormal system in $L^{2}((\delta-r, \delta+r) \times \mathbb{R})$. Since $\lambda \mapsto \widehat{\Theta y}(\lambda)$ is analytic in $\{\operatorname{Re} \lambda>0\}$ for all $y \in F$, the mean value property 
for harmonic functions implies that

$$
\frac{1}{\sqrt{\pi r^{2}}} \iint_{(\delta-r, \delta+r) \times \mathbb{R}} f_{n}^{\rho}(s, t) \widehat{\Theta y}(s+i t) d s d t=\widehat{\Theta y}(\delta+2 i(n+\rho) r) .
$$

Let us write $F_{y}(s, t):=\widehat{\Theta y}(s+i t)$. Applying $(2.1)$ to the operator $\mathscr{F}: L^{2}\left(\mathbb{R}_{+}\right) \rightarrow$ $L^{2}((\delta-r, \delta+r) \times \mathbb{R})$ defined by

$$
(\mathscr{F} f)(\lambda, \mu)=\int_{0}^{\infty} e^{-(\lambda+i \mu) t} f(t) d t, \quad f \in L^{1}\left(\mathbb{R}_{+}\right) \cap L^{2}\left(\mathbb{R}_{+}\right),
$$

and noting that $\widetilde{\mathscr{F}}(\Theta y)$ is represented by $F_{y}$, we obtain

$$
\begin{aligned}
\mathbb{E} & \left\|\sum_{n=-N}^{N} g_{n} \widehat{\Theta y}(\delta+2 i(n+\rho) r)\right\|^{2} \\
& =\frac{1}{\pi r^{2}} \mathbb{E}\left\|\sum_{n=-N}^{N} g_{n} \iiint_{(\delta-r, \delta+r) \times \mathbb{R}} f_{n}^{\rho}(s, t) F_{y}(s, t) d s d t\right\|^{2} \\
& \leqslant \frac{1}{\pi r^{2}}\left\|F_{y}\right\|_{\gamma((\delta-r, \delta+r) \times \mathbb{R} ; E)}^{2} \stackrel{(*)}{\leqslant} \frac{4}{r}\|\Theta y\|_{\gamma\left(L^{2}\left(\mathbb{R}_{+}\right), E\right)}^{2} \stackrel{(* *)}{\leqslant} 16\|\Theta\|^{2} \max \left\{1, \frac{1}{\delta}\right\}\|y\|^{2} .
\end{aligned}
$$

In $(*)$ we used the estimate $\|\mathscr{F}\|^{2} \leqslant 4 \pi r$ and in $(* *)$ the choice of $r$. By Lemma 3.2 , the sequence $(\widehat{\Theta}(\delta+2 i(n+\rho) r))_{n \in \mathbb{Z}}$ is $R$-bounded, uniformly with respect to $\rho \in[0,1)$, with an $R$-bound of order $C_{\Theta} \max \{1,1 / \sqrt{\delta}\}$.

For $0<\delta<1$, by a scaling argument we may apply Lemma 3.3 with $\eta=\frac{1}{2}$ to the points $\delta+i(n+\rho) \delta$ (for $k=0$; this corresponds to the choice $r=\frac{1}{2} \delta$ ) and $2 \delta+i(n+\rho) \delta$ (for $k=1$; this corresponds to the choice $r=\frac{1}{4} \delta$ ). We obtain that $\widehat{\Theta}$ is $R$-bounded on the vertical line $\left\{\operatorname{Re} \lambda=\frac{3}{2} \delta\right\}$ with an $R$-bound of order $\|\Theta\| / \sqrt{\delta}$.

Similarly, for $\delta \geqslant 1$ we apply Lemma 3.3 with $\eta=\frac{1}{2}$ to the points $\delta+i(n+\rho)$ and $\delta+1+i(n+\rho)$ (for $k=0,1$; this corresponds to $r=\frac{1}{2}$ ). We obtain that $\widehat{\Theta}$ is $R$-bounded on the vertical line $\left\{\operatorname{Re} \lambda=\delta+\frac{1}{2}\right\}$ with an $R$-bound of order $\|\Theta\|$.

Now let $\delta>0$ be fixed again and consider, for $\varepsilon>0$, the strip $S_{\delta, \varepsilon}=\{\delta \leqslant$ $\operatorname{Re} \lambda \leqslant \varepsilon\}$. By the above, $\widehat{\Theta}$ is $R$-bounded on $\partial S_{\delta, \varepsilon}$ with an $R$-bound of order $\|\Theta\| \max \{1,1 / \sqrt{\delta}\}$. By [32, Proposition 2.8], $\widehat{\Theta}$ is $R$-bounded on $S_{\delta, \varepsilon}$ with the same $R$-bound.

If $E$ has property $\left(\alpha^{+}\right)$, a considerably simpler proof of this result can be based upon [26, Theorem 6.5].

\section{INVARIANT MEASURES}

In this section we return to the problem $\left(\mathrm{SCP}_{B}\right)$ and discuss existence and uniqueness of solutions and their asymptotic behaviour. Throughout this section, $A$ is the generator of a $C_{0}$-semigroup on $E, H$ is a separable real Hilbert space, and $B \in \mathscr{L}(H, E)$ is a fixed bounded operator.

A cylindrical $H$-Brownian motion on a probability space $(\Omega, \mathscr{F}, \mathbb{P})$ is a family $\mathbb{W}_{H}=\left\{W_{H}(t)\right\}_{t \in[0, T]}$ bounded linear operators from $H$ into $L^{2}(\Omega)$ with the following properties:

(1) For all $h \in H,\left\{W_{H}(t) h\right\}_{t \in[0, T]}$ is a standard Brownian motion;

(2) For all $s, t \in[0, T]$ and $g, h \in H, \mathbb{E}\left(W_{H}(s) g \cdot W_{H}(t) h\right)=(s \wedge t)[g, h]_{H}$. 
We shall always assume that the Brownian motions $W_{H} h$ are adapted to some given filtration.

An $E$-valued process $U=\{U(t)\}_{t \geqslant 0}$ on $(\Omega, \mathscr{F}, \mathbb{P})$ is called a weak solution of the problem $\left(\mathrm{SCP}_{B}\right)$ if it is weakly progressively measurable and for all $x^{*} \in \mathscr{D}\left(A^{*}\right)$, the domain of the adjoint operator $A^{*}$, the following two conditions are satisfied:

(1) Almost surely, the paths $t \mapsto\left\langle U(t), A^{*} x^{*}\right\rangle$ are locally integrable;

(2) For all $t \geqslant 0$ we have, almost surely,

$$
\left\langle U(t), x^{*}\right\rangle=\int_{0}^{t}\left\langle U(s), A^{*} x^{*}\right\rangle d s+W_{H}(t) B^{*} x^{*} .
$$

To simplify terminology we shall simply speak of a solution. The following result from [24] gives necessary and sufficient conditions for existence (and uniqueness) of solutions; see also $[4,6]$.

Proposition 4.1. The following assertions are equivalent:

(1) The function $t \mapsto S(t) B$ belongs to $\gamma(0, T ; H, E)$ for some $T>0$;

(2) The function $t \mapsto S(t) B$ belongs to $\gamma(0, T ; H, E)$ for all $T>0$;

(3) The problem $\left(\mathrm{SCP}_{B}\right)$ admits a solution $U$.

The solution $U$ is unique up to a modification and Gaussian. The covariance operator $Q_{t} \in \mathscr{L}\left(E^{*}, E\right)$ of $U(t)$ is given by

$$
\mathbb{E}\left\langle U(t), x^{*}\right\rangle^{2}=\left\langle Q_{t} x^{*}, y^{*}\right\rangle=\int_{0}^{t}\left\langle S(s) B B^{*} S^{*}(s) x^{*}, y^{*}\right\rangle d s, \quad x^{*}, y^{*} \in E^{*}, t \geqslant 0 .
$$

Moreover,

$$
\mathbb{E}\|U(t)\|^{2}=\|S \circ B\|_{\gamma(0, t ; H, E)}^{2}, \quad t \geqslant 0 .
$$

In combination with Anderson's inequality, it follows from this proposition that the problem $\left(\mathrm{SCP}_{B}\right)$ has a solution if and only if it has a solution with $A$ replaced by the rescaled operator $A-\omega$.

If $U$ is a solution of $\left(\mathrm{SCP}_{B}\right)$, its transition semigroup on the space $B_{b}(E)$ of all real-valued bounded Borel functions on $E$ is defined by

$$
(P(t) f)(x)=\mathbb{E}(f(S(t) x+U(t))), \quad t \geqslant 0, x \in E, f \in B_{b}(E) .
$$

A Radon measure $\mu$ on $E$ is said to be invariant under the semigroup $P=\{P(t)\}_{t \geqslant 0}$ if for all $f \in B_{b}(E)$ and $t \geqslant 0$ we have

$$
\int_{E} P(t) f d \mu=\int_{E} f d \mu .
$$

The following two propositions, 4.2 and 4.4, extend corresponding Hilbert space results in $[7$, Chapter 6$]$.

Proposition 4.2. Assume that the problem $\left(\mathrm{SCP}_{B}\right)$ admits a solution, and let $\mu$ be a Radon probability measure on E. The following assertions are equivalent:

(1) $\mu$ is is an invariant measure for $\left(\mathrm{SCP}_{B}\right)$;

(2) (i) The weak operator limit $Q_{\infty}=\lim _{t \rightarrow \infty} Q_{t}$ exists in $\mathscr{L}\left(E^{*}, E\right)$ and is the covariance of a centred Gaussian Radon measure $\mu_{\infty}$ on $E$,

(ii) We have $\mu=\nu * \mu_{\infty}$, where $\nu$ is an invariant measure for $S$.

Moreover, $\mu_{\infty}$ is an invariant measure for $\left(\mathrm{SCP}_{B}\right)$. 
Explicitly, $\nu$ is a Radon probability measure on $E$ which satisfies, for all $f \in$ $B_{b}(E)$ and $t \geqslant 0$,

$$
\int_{E} f(S(t) x) d \nu(x)=\int_{E} f(x) d \nu(x) .
$$

For the reader's convenience we sketch the proof of the implication $(1) \Rightarrow(2)$; the converse implication is obvious.

Proof of $(1) \Rightarrow(2)$. Taking $f(x)=\exp \left(-i\left\langle x, x^{*}\right\rangle\right)$ in (4.1) we obtain, for all $x^{*} \in E^{*}$ and $t \geqslant 0$,

$$
\begin{aligned}
\exp \left(-\frac{1}{2}\left\langle Q_{t} x^{*}, x^{*}\right\rangle\right) \widehat{\mu}\left(S^{*}(t) x^{*}\right) & =\mathbb{E} \exp \left(-i\left\langle U(t), x^{*}\right\rangle \widehat{\mu}\left(S^{*}(t) x^{*}\right)\right. \\
& =\int_{E} \mathbb{E} \exp \left(-i\left\langle S(t) x+U(t), x^{*}\right\rangle\right) d \mu(x) \\
& =\int_{E} \mathbb{E} \exp \left(-i\left\langle x, x^{*}\right\rangle\right) d \mu(x)=\widehat{\mu}\left(x^{*}\right) .
\end{aligned}
$$

If $\widehat{\mu}\left(x^{*}\right) \neq 0$, then $\widehat{\mu}\left(S^{*}(t) x^{*}\right) \neq 0$ and

$$
\exp \left(-\frac{1}{2}\left\langle Q_{t} x^{*}, x^{*}\right\rangle\right)=\left|\frac{\widehat{\mu}\left(x^{*}\right)}{\widehat{\mu}\left(S^{*}(t) x^{*}\right)}\right| \geqslant\left|\widehat{\mu}\left(x^{*}\right)\right| .
$$

On the other hand, $t \mapsto\left\langle Q_{t} x^{*}, x^{*}\right\rangle$ is nondecreasing. It follows that the limit $q_{\infty}\left(x^{*}\right):=\lim _{t \rightarrow \infty}\left\langle Q_{t} x^{*}, x^{*}\right\rangle$ exists and is finite. This, in turn, implies that the limit $n\left(x^{*}\right):=\lim _{t \rightarrow \infty} \widehat{\mu}\left(S^{*}(t) x^{*}\right)$ exists, and we obtain the identity

$$
\exp \left(-\frac{1}{2} q_{\infty}\left(x^{*}\right)\right) n\left(x^{*}\right)=\widehat{\mu}\left(x^{*}\right) .
$$

If $\widehat{\mu}\left(x^{*}\right)=0$, then $\widehat{\mu}\left(S^{*}(t) x^{*}\right)=0$ for all $t \geqslant 0$ and we put $n\left(x^{*}\right):=0$. Also, $q_{\infty}\left(c x^{*}\right) \neq 0$ for $c>0$ sufficiently small, and we put $q_{\infty}\left(x^{*}\right):=c^{-2} q_{\infty}\left(c x^{*}\right)$. In this way, (4.2) extends to all $x^{*} \in E^{*}$. Moreover, the functions $x^{*} \mapsto n\left(x^{*}\right)$ and $x^{*} \mapsto r\left(x^{*}\right):=\exp \left(-\frac{1}{2} q_{\infty}\left(x^{*}\right)\right)$ are positive definite in the sense that

$$
\sum_{i, j=1}^{n} c_{i} \overline{c_{j}} n\left(x_{i}^{*}-x_{j}^{*}\right) \geqslant 0 \text { and } \sum_{i, j=1}^{n} c_{i} \overline{c_{j}} r\left(x_{i}^{*}-x_{j}^{*}\right) \geqslant 0
$$

for all finite sequences $c_{1}, \ldots, c_{n} \in \mathbb{C}$ and $x_{1}^{*}, \ldots, x_{n}^{*} \in E^{*}$, and pseudocontinuous in the sense that their restrictions to any finite-dimensional subspace of $E^{*}$ are continuous. Also, $r$ is symmetric in the sense that $r\left(x^{*}\right)=r\left(-x^{*}\right)$ for all $x^{*} \in E^{*}$. Hence by [31, Proposition VI.3.2], $n$ and $r$ are the Fourier transforms of cylindrical measures $\nu$ and $\mu_{\infty}$ on $E$. Clearly, $\nu * \mu_{\infty}=\mu$ as cylindrical measures. Since $\mu$ is a Radon measure on $E$, it follows from [31, Proposition VI.3.4] that $\nu$ and $\mu_{\infty}$ have Radon extensions as well. In view of

$$
\widehat{\nu}\left(S^{*}(s) x^{*}\right)=n\left(S^{*}(s) x^{*}\right)=\lim _{t \rightarrow \infty} \widehat{\mu}\left(S^{*}(t+s) x^{*}\right)=n\left(x^{*}\right)=\widehat{\nu}\left(x^{*}\right),
$$

the measure $\nu$ is invariant under $S$. The measure $\mu_{\infty}$ is Gaussian, and its covariance operator $Q_{\infty}$ is given by $\left\langle Q_{\infty} x^{*}, x^{*}\right\rangle=q_{\infty}\left(x^{*}\right)$. The proof that $\mu_{\infty}$ is invariant is standard.

In general an invariant measure, if it exists, is not unique. A simple sufficient condition for uniqueness is stated in the following result, which is closely related to [23, Corollary 2.13]. 
Corollary 4.3. Assume that the problem $\left(\mathrm{SCP}_{B}\right)$ admits a solution. If there exists a weak ${ }^{*}$-sequentially dense subspace $F$ of $E^{*}$ such that weak ${ }^{*}-\lim _{t \rightarrow \infty} S^{*}(t) x^{*}=0$ for all $x^{*} \in F$, then $\left(\mathrm{SCP}_{B}\right)$ admits at most one invariant measure.

Proof. Suppose an invariant measure $\mu$ exists; we shall prove that $\mu=\mu_{\infty}$ by showing that $\nu=\delta_{0}$.

Since $\nu$ is invariant for $S$, for all $x^{*} \in E^{*}$ and $t \geqslant 0$ we have

$$
\int_{E} \exp \left(-i\left\langle S(t) x, x^{*}\right\rangle\right) d \nu(x)=\int_{E} \exp \left(-i\left\langle x, x^{*}\right\rangle\right) d \nu(x),
$$

or equivalently, $\hat{\nu}\left(S^{*}(t) x^{*}\right)=\hat{\nu}\left(x^{*}\right)$. By the dominated convergence theorem, for all $x^{*} \in F$ we obtain

$$
\hat{\nu}\left(x^{*}\right)=\lim _{t \rightarrow \infty} \hat{\nu}\left(S^{*}(t) x^{*}\right)=\hat{\nu}(0)=1 .
$$

Since $F$ is weak ${ }^{*}$-sequentially dense in $E^{*}$, another application of the dominated convergence theorem shows that $\hat{\nu}\left(x^{*}\right)=1$ for all $x^{*} \in E^{*}$. Hence $\nu=\delta_{0}$ as claimed.

The assumption on $S$ is satisfied if the resolvent $R(\lambda, A)$ is uniformly bounded on $\{\operatorname{Re} \lambda>0\}$. To see this, let $A^{\odot}$ denote the part of $A^{*}$ in $E^{\odot}:=\overline{\mathscr{D}\left(A^{*}\right)}$. The restriction $S^{\odot}:=\left.S^{*}\right|_{E \odot}$ is strongly continuous on $E^{\odot}$ and its generator is $A^{\odot}$. Also, $R\left(\lambda, A^{\odot}\right)$ is uniformly bounded on $\{\operatorname{Re} \lambda>0\}$. An elementary stability result for $C_{0}$-semigroups due to Slemrod [30] then implies that $\lim _{t \rightarrow \infty} S^{\odot}(t) x^{\odot}=0$ strongly for all $x^{\odot} \in \mathscr{D}\left(\left(A^{\odot}\right)^{2}\right)$ (by [33] this actually holds for all $x^{\odot} \in \mathscr{D}\left(A^{\odot}\right)$ ). Note that $\mathscr{D}\left(\left(A^{\odot}\right)^{2}\right)$ is indeed weak ${ }^{*}$-sequentially dense in $E^{*}$.

The following proposition describes the precise relationship between the spaces $\gamma(0, T ; H, E)$, the existence of solutions for $\left(\mathrm{SCP}_{B}\right)$ and their asymptotic behaviour.

Proposition 4.4. The following assertions are equivalent:

(1) The function $t \mapsto S(t) B$ belongs to $\gamma(0, T ; H, E)$ for all $T>0$ and

$$
\sup _{T>0}\|S \circ B\|_{\gamma(0, T ; H, E)}<\infty ;
$$

(2) The problem $\left(\mathrm{SCP}_{B}\right)$ admits a weak solution which is bounded in probability. Also, the following assertions are equivalent:

$\left(1^{\prime}\right)$ The function $t \mapsto S(t) B$ belongs to $\gamma\left(\mathbb{R}_{+} ; H, E\right)$;

$\left(2^{\prime}\right)$ The problem $\left(\mathrm{SCP}_{B}\right)$ admits an invariant measure.

Furthermore, $\left(1^{\prime}\right)$ and $\left(2^{\prime}\right)$ imply (1) and (2), and all four assertions are equivalent if $E$ does not contain an isomorphic copy of $c_{0}$.

Proof. The proof is a routine generalization of the corresponding Hilbert space results in $[6,7]$, modulo some subtle points involving the geometry of Banach spaces. For the convenience of the reader we spell out the details.

$(1) \Rightarrow(2)$ : Let $U$ be a weak solution of the problem $\left(\mathrm{SCP}_{B}\right)$. For $t \geqslant 0$ let $\mu_{t}$ denote the distribution of the random variable $U(t)$. By Chebyshev's inequality we have

$$
\mathbb{P}(\|U(t)\|>r) \leqslant \frac{1}{r^{2}} \int_{E}\|x\|^{2} d \mu_{t}(x)=\frac{1}{r^{2}}\|S \circ B\|_{\gamma(0, t ; H, E)}^{2},
$$

where we used the identity in Proposition 4.1. Since by assumption we have $\sup _{t>0}\|S \circ B\|_{\gamma(0, t ; H, E)}<\infty$ it follows that $U$ is bounded in probability. 
$(2) \Rightarrow(1)$ : As in $[7$, Theorem 6.2.3] this follows from Fernique's theorem [6, Theorem 2.6].

$\left(1^{\prime}\right) \Rightarrow\left(2^{\prime}\right)$ : By assumption, the $\mathscr{L}(H, E)$-valued function $S \circ B$ represents the operator $R \in \gamma\left(L^{2}\left(\mathbb{R}_{+} ; H\right), E\right)$ given by

$$
\left\langle R f, x^{*}\right\rangle=\int_{0}^{\infty}\left[B^{*} S^{*}(t) x^{*}, f(t)\right]_{H} d t, \quad f \in L^{2}\left(\mathbb{R}_{+} ; H\right), x^{*} \in E^{*} .
$$

By direct computation, $R R^{*}$ satisfies

$$
\left\langle R R^{*} x^{*}, y^{*}\right\rangle=\int_{0}^{\infty}\left\langle S(t) B B^{*} S^{*}(t) x^{*}, y^{*}\right\rangle d t, \quad x^{*}, y^{*} \in E^{*} .
$$

By Proposition 4.2 the centred Gaussian measure on $E$ with covariance operator $R R^{*}$ is an invariant measure for $\left(\mathrm{SCP}_{B}\right)$.

$\left(2^{\prime}\right) \Rightarrow\left(1^{\prime}\right)$ : Let $\mu_{\infty}$ be the invariant measure with covariance operator $Q_{\infty}$ as defined in Proposition 4.2. We have

$$
\left\langle Q_{\infty} x^{*}, x^{*}\right\rangle=\int_{0}^{\infty}\left\langle S(t) B B^{*} S^{*}(t) x^{*}, x^{*}\right\rangle d t=\int_{0}^{\infty}\left\|B^{*} S^{*}(t) x^{*}\right\|_{H}^{2} d t,
$$

which shows that $B^{*} S^{*}(\cdot) x^{*}$ belongs to $L^{2}\left(\mathbb{R}_{+} ; H\right)$. Hence we may define a bounded operator $R: L^{2}\left(\mathbb{R}_{+} ; H\right) \rightarrow E^{* *}$ by

$$
\left\langle x^{*}, R f\right\rangle:=\int_{0}^{\infty}\left[B^{*} S^{*}(t) x^{*}, f(t)\right]_{H} d t, \quad f \in L^{2}\left(\mathbb{R}_{+} ; H\right), x^{*} \in E^{*} .
$$

If $f \in L^{2}\left(\mathbb{R}_{+} ; H\right)$ is supported in an interval $[0, r]$, then

$$
R f=\int_{0}^{r} S(t) B f(t) d t
$$

where the integral exists as a Bochner integral in $E$. Since the functions with bounded support are dense in $L^{2}\left(\mathbb{R}_{+} ; H\right)$ it follows that $R$ takes values in $E$. Hence $R$ is represented by $S \circ B$, and since $R \circ R^{*}=Q_{\infty}$ is a Gaussian covariance this implies that $S \circ B \in \gamma\left(\mathbb{R}_{+} ; H, E\right)$.

$\left(1^{\prime}\right) \Rightarrow(1)$ : This is immediate from the ideal property.

Finally assume that $E$ does not contain a copy of $c_{0}$.

$(1) \Rightarrow\left(1^{\prime}\right)$ : As in $[18$, Lemma 4.10] this follows from Fatou's lemma in combination with a theorem of Hoffmann-Jørgensen and Kwapień [21, Theorem 9.29].

The assumption that $E$ should not contain a copy of $c_{0}$ cannot be omitted from the final assertion of the proposition. As a consequence we see that the problem $\left(\mathrm{SCP}_{B}\right)$ may fail to admit an invariant measure even if a solution exists which is bounded in probability. This is shown by the following example, in which the operator $B$ is of rank 1 .

Example 9. Let $\varphi:[0, \infty) \rightarrow \mathbb{R}_{+}$be a $C^{1}$-function with compact support in $(0,1)$ such that $\|\varphi\|_{2}=1$ and define

$$
\phi(t):=\sum_{n \geqslant 1} \varphi(t-n) x_{n},
$$

where $x_{n} \in c_{0}$ is the seqence

$$
x_{n}=(0, \ldots, 0,1 / \sqrt{\ln (n+1)}, 0, \ldots) .
$$


We claim that the function $\phi$ does not belong to $\gamma\left(\mathbb{R}_{+} ; c_{0}\right)$. To see this, note that

$$
\int_{0}^{\infty}\left\langle\phi(t), e_{n}^{*}\right\rangle^{2} d t=\frac{1}{\ln (n+1)} \int_{n}^{n+1} \varphi^{2}(t-n) d t=\frac{1}{\ln (n+1)},
$$

where $e_{n}^{*}=(0, \ldots, 0,1,0 \ldots)$ is the $n$-th unit vector of $c_{0}^{*}=l^{1}$. Hence,

$$
\int_{0}^{\infty}\left\langle\phi(t), x^{*}\right\rangle^{2} d t=\left\langle Q x^{*}, x^{*}\right\rangle \quad \forall x^{*} \in l^{1},
$$

where $Q \in \mathscr{L}\left(l^{1}, c_{0}\right)$ is given by $Q\left(\left(\alpha_{n}\right)_{n \geqslant 1}\right):=\left(\alpha_{n} / \ln (n+1)\right)_{n \geqslant 1}$. It is shown in $[20$, Theorem 11] that this operator is not a Gaussian covariance and it follows that $\phi \notin \gamma\left(\mathbb{R}_{+} ; c_{0}\right)$ as claimed. By the same argument, [20, Theorem 11] further shows that for all $T>0$ we have $\phi \in \gamma\left(0, T ; c_{0}\right)$ and

$$
\sup _{T>0}\|\phi\|_{\gamma\left(0, T ; c_{0}\right)}<\infty .
$$

Let $E:=B U C\left([0, \infty) ; c_{0}\right)$ denote the Banach space of all bounded and uniformly continuous functions $f:[0, \infty) \rightarrow c_{0}$. It is easily checked that the function $\phi$ constructed above belongs to $E$. Let $S$ denote the left translation semigroup on $E$, $S(t) f(s)=f(t+s)$.

Since $\phi$ is $C^{1}$, for all $s \geqslant 0$ this function is stochastically integrable with respect to the Brownian motion defined by $W_{s}(t):=W(s+t)-W(s)$, and an integration by parts gives

$$
\begin{aligned}
\int_{0}^{T} \phi(s+t) d W_{s}(t) & =\phi(s+T) W_{s}(T)-\int_{0}^{T} \phi^{\prime}(s+t) W_{s}(t) d t \\
& =\phi(s+T) W(s+T)-\phi(s) W(s)-\int_{s}^{s+T} \phi^{\prime}(t) W(t) d t \\
& =\int_{s}^{s+T} \phi(t) d W(t) .
\end{aligned}
$$

The $E$-valued function $S \phi$, being $C^{1}$ as well, belongs to $\gamma(0, T ; E)$. Evaluating its $\gamma$-norm of by means of the second moment of its stochastic integral, with (4.5) and Doob's maximal inequality we obtain

$$
\begin{aligned}
\|S \phi\|_{\gamma(0, T ; E)} & =\left(\mathbb{E}\left\|\int_{0}^{T} S(t) \phi d W(t)\right\|_{E}^{2}\right)^{\frac{1}{2}} \\
& =\left(\mathbb{E} \sup _{s \geqslant 0}\left\|\int_{0}^{T} \phi(s+t) d W(t)\right\|_{c_{0}}^{2}\right)^{\frac{1}{2}} \\
& =\left(\mathbb{E} \sup _{s \geqslant 0}\left\|\int_{0}^{T} \phi(s+t) d W_{s}(t)\right\|_{c_{0}}^{2}\right)^{\frac{1}{2}} \\
& =\left(\mathbb{E} \sup _{s \geqslant 0}\left\|\int_{s}^{s+T} \phi(t) d W(t)\right\|_{c_{0}}^{2}\right)^{\frac{1}{2}} \\
& \leqslant 2\left(\mathbb{E} \sup _{r \geqslant 0}\left\|\int_{0}^{r} \phi(t) d W(t)\right\|_{c_{0}}^{2}\right)^{\frac{1}{2}} \\
& \leqslant 4 \sup _{r \geqslant 0}\left(\mathbb{E}\left\|\int_{0}^{r} \phi(t) d W(t)\right\|_{c_{0}}^{2}\right)^{\frac{1}{2}} \leqslant 4 \sup _{r \geqslant 0}\|\phi\|_{\gamma\left(0, r ; c_{0}\right)} .
\end{aligned}
$$

With (4.4) it follows that $\sup _{T>0}\|S \phi\|_{\gamma(0, T ; E)}<\infty$ and the claim is proved. 
Next we check that $S \phi \notin \gamma\left(\mathbb{R}_{+} ; E\right)$. Let $\delta_{0}: E \rightarrow c_{0}$ be defined by $\delta_{0} f:=f(0)$. Then $\left\langle S(t) \phi, \delta_{0}\right\rangle=\phi(t)$ for all $t \geqslant 0$, which implies that $\left\langle S \phi, \delta_{0}\right\rangle=\phi \notin \gamma\left(\mathbb{R}_{+} ; c_{0}\right)$. Therefore, $S \phi \notin \gamma\left(\mathbb{R}_{+} ; E\right)$ as claimed.

This example shows that the implication $(1) \Rightarrow\left(1^{\prime}\right)$ of Proposition 4.4 fails for the semigroup $S$ on $E=B U C\left([0, \infty) ; c_{0}\right)$ if we take $H=\mathbb{R}$ and define $B: \mathbb{R} \rightarrow E$ by $B t:=t \phi$.

The content of the following proposition is that $\left(\mathrm{SCP}_{B}\right)$ admits a unique invariant measure whenever $\left(\mathrm{SCP}_{B}\right)$ admits a solution and the semigroup generated by $A$ is uniformly exponentially stable. It can be thought of as a preliminary version of Theorem 1.1.

Proposition 4.5. Let $T>0$ and $B \in \mathscr{L}(H, E)$ be fixed. If the function $t \mapsto S(t) B$ belongs to $\gamma(0, T ; H, E)$, then for all $\omega>\omega_{0}(A)$ the function $t \mapsto e^{-\omega t} S(t) B$ belongs to $\gamma\left(\mathbb{R}_{+} ; H, E\right)$.

Proof. First we note that by the semigroup property and the ideal property, $t \mapsto$ $S(t) B$ belongs to $\gamma(0, T ; H, E)$ for all $T>0$; cf. [24, Corollary 7.2]. Choose $t_{0}>0$ large enough such that $e^{-\omega t_{0}}\left\|S\left(t_{0}\right)\right\|<1$. By the ideal property, the operators $V_{n}$ defined by

$$
V_{n} f:=\int_{n t_{0}}^{(n+1) t_{0}} e^{-\omega t} S(t) B f(t) d t, \quad n \in \mathbb{N}, f \in L^{2}\left(\mathbb{R}_{+} ; H\right),
$$

belong to $\gamma\left(L^{2}\left(\mathbb{R}_{+} ; H\right), E\right)$. We have $V_{n} f=e^{-\omega n t_{0}} S\left(n t_{0}\right) V_{0} T_{n} f$, where $T_{n}$ is the left translation operator over $n t_{0}$, i.e., $T_{n} f(t):=f\left(t+n t_{0}\right)$ for $t \in \mathbb{R}_{+}$and $f \in$ $L^{2}\left(\mathbb{R}_{+} ; H\right)$. Writing $\|\cdot\|_{\gamma}:=\|\cdot\|_{\gamma\left(L^{2}\left(\mathbb{R}_{+} ; H\right), E\right)}$, it follows from the ideal property that

$$
\left\|V_{n}\right\|_{\gamma} \leqslant e^{-\omega n t_{0}}\left\|S\left(n t_{0}\right)\right\|\left\|V_{0}\right\|_{\gamma}\left\|T_{n}\right\| \leqslant e^{-\omega n t_{0}}\left\|S\left(t_{0}\right)\right\|^{n}\left\|V_{0}\right\|_{\gamma} .
$$

Since $e^{-\omega t_{0}}\left\|S\left(t_{0}\right)\right\|<1$ it follows that $\sum_{n \geqslant 0}\left\|V_{n}\right\|_{\gamma}<\infty$. By the completeness of $\gamma\left(L^{2}\left(\mathbb{R}_{+} ; H\right), E\right)$, the sum $\sum_{n \geqslant 0} V_{n}$ converges absolutely to some operator $V \in$ $\gamma\left(L^{2}\left(\mathbb{R}_{+} ; H\right), E\right)$. This operator is represented by $t \mapsto e^{-\omega t} S(t) B$, and therefore $t \mapsto e^{-\omega t} S(t) B$ belongs to $\gamma\left(\mathbb{R}_{+} ; H, E\right)$.

By combining the propositions and considering the special case $H=\mathbb{R}$ in the second statement, we obtain the following result.

Corollary 4.6. The following assertions hold.

(1) We have $\omega_{\text {inv }}^{B}(A)<\infty$ if and only if $\left(\mathrm{SCP}_{B}\right)$ admits a solution, in which case $\omega_{\mathrm{inv}}^{B}(A) \leqslant \omega_{0}(A)$;

(2) We have $\omega_{\text {inv }}^{(1)}(A)<\infty$ if and only if $\left(\mathrm{SCP}_{B}\right)$ admits a solution for all rank 1 operators $B \in \mathscr{L}(H, E)$, in which case $\omega_{\text {inv }}^{(1)}(A) \leqslant \omega_{0}(A)$;

(3) We have $\omega_{\mathrm{inv}}^{\gamma}(A)<\infty$ if and only if $\left(\mathrm{SCP}_{B}\right)$ admits a solution for all $\gamma$-radonifying operators $B \in \mathscr{L}(H, E)$, in which case $\omega_{\text {inv }}^{\gamma}(A) \leqslant \omega_{0}(A)$.

To conclude this section we prove a result which relates the existence of an invariant measure to the moments of the solution. Define, for $p \in[1, \infty)$,

$$
\begin{aligned}
\omega_{p}^{B}(A)=\inf \{\omega \in \mathbb{R}: & \text { the problem }\left(\mathrm{SCP}_{B}\right) \text { with } A \text { replaced by } A-\omega \\
& \text { has a solution } \left.U_{\omega} \text { which satisfies } \sup _{t \geqslant 0} \mathbb{E}\left\|U_{\omega}(t)\right\|^{p}<\infty\right\} .
\end{aligned}
$$


Proposition 4.7. If the problem $\left(\mathrm{SCP}_{B}\right)$ admits a solution, then for all $p \in[1, \infty)$ we have $\omega_{\mathrm{inv}}^{B}(A)=\omega_{p}^{B}(A)$.

Proof. Let $p \in[1, \infty)$ be fixed.

If $\omega_{\text {inv }}^{B}(A)<c$, then the problem $\left(\mathrm{SCP}_{B}\right)$ with $A$ replaced by $A_{c}:=A-c$ admits an invariant measure $\mu_{c, \infty}$ whose convariance operator $Q_{c, \infty}$ is given as in (4.3). Denote the solution of $\left(\mathrm{SCP}_{B}\right)$ by $U_{c}$ and let $\mu_{t, c}$ be the distribution of $U_{c}(t)$. By Anderson's inequality and general convergence results for Gaussian measures [3, Chapter 3] we have

$$
\sup _{t \geqslant 0} \mathbb{E}\left\|U_{c}(t)\right\|^{p}=\lim _{t \rightarrow \infty} \mathbb{E}\left\|U_{c}(t)\right\|^{p}=\lim _{t \rightarrow \infty} \int_{E}\|x\|^{p} d \mu_{c, t}(x)=\int_{E}\|x\|^{p} d \mu_{c, \infty}(x) .
$$

The right hand side is finite by Fernique's theorem. Accordingly we find that $\omega_{p}^{B}(A) \leqslant c$. This proves the inequality $\omega_{p}^{B}(A) \leqslant \omega_{\text {inv }}^{B}(A)$.

If $\omega_{p}^{B}(A)<c$, then the solution of $\left(\mathrm{SCP}_{B}\right)$ with $A$ replaced by $A_{c}$ is bounded in probability, and therefore Proposition 4.4 shows that $\sup _{t \geqslant 0}\left\|S_{c} \circ B\right\|_{\gamma(0, t ; H, E)}<\infty$. Arguing as in Proposition 4.5 we obtain from this that $S_{c^{\prime}} \circ B \in \gamma\left(\mathbb{R}_{+} ; H, E\right)$ for all $c^{\prime}>c$. Another application of Proposition 4.4 then shows that $\omega_{\text {inv }}^{B}(A) \leqslant c$. This proves the inequality $\omega_{\mathrm{inv}}^{B}(A) \leqslant \omega_{p}^{B}(A)$.

\section{Proofs of the Main theorems}

We now turn to the proofs of the theorems stated in the introduction.

Lemma 5.1. The following assertions are equivalent:

(1) The function $t \mapsto e^{-\omega t} S(t) B$ belongs to $\gamma\left(\mathbb{R}_{+} ; H, E\right)$;

(2) The function $t \mapsto R(\omega+i t, A) B$ belongs to $\gamma(\mathbb{R} ; H, E)$.

In this situation we have

$$
\left\|e^{-\omega(\cdot)} S(\cdot) B\right\|_{\gamma\left(\mathbb{R}_{+} ; H, E\right)}^{2}=\frac{1}{2 \pi}\|R(\omega+i(\cdot), A) B\|_{\gamma(\mathbb{R} ; H, E)}^{2} .
$$

Proof. Apply (2.1) to the Fourier-Plancherel transform on $L^{2}(\mathbb{R} ; H)$.

Proof of Theorem 1.1. The proof is divided into two steps.

Step 1 - First we show that $s_{\gamma}(A)<0$. Let $\Gamma:=\gamma(\mathscr{R})$ denote the $\gamma$-bound of the family $\mathscr{R}:=\{R(\lambda, A): \operatorname{Re} \lambda>0\}$ and put $\delta:=1 / \Gamma$. Since $\|R(\lambda, A)\| \leqslant \Gamma$ for all $\operatorname{Re} \lambda>0$, standard arguments from spectral theory imply that $S_{\delta}:=\{\lambda \in \mathbb{C}$ : $-\delta<\operatorname{Re} \lambda<\delta\} \subseteq \varrho(A)$ and

$$
R(\lambda, A)=\sum_{n \geqslant 0}(-\operatorname{Re} \lambda)^{n} R(i \operatorname{Im} \lambda, A)^{n+1}, \quad \forall \lambda \in S_{\delta} .
$$

By (3.2) the set $\{R(i t, A): t \in \mathbb{R}\}$ is $\gamma$-bounded with $\gamma$-bound $\Gamma$. Hence by (3.1) the family $\left\{R(\lambda, A): \lambda \in S_{\frac{1}{2} \delta}\right\}$ is $\gamma$-bounded with $\gamma$-bound $2 \Gamma$. It follows that $s_{\gamma}(A) \leqslant-\frac{1}{2} \delta$.

Step 2 - Now we turn to the actual proof of the theorem.

We shall prove that the orbit $t \mapsto S(t) B$ belongs to $\gamma\left(\mathbb{R}_{+} ; H, E\right)$. The existence of an invariant measure then follows from Proposition 4.4. Its uniqueness follows from Corollary 4.3, the remark following it, and the fact that $R$-boundedness implies uniform boundedness. 
Fix $s_{\gamma}(A)<\zeta<0$ and $\omega>\omega_{0}(A)$. The rescaled orbit $t \mapsto e^{-\omega t} S(t) B$ belongs to $\gamma\left(\mathbb{R}_{+} ; H, E\right)$ by Proposition 4.5 , which applies thanks to Proposition 4.1. By Lemma $5.1, t \mapsto R(\omega+i t, A) B$ belongs to $\gamma(\mathbb{R} ; H, E)$.

Let $\gamma\left(\mathscr{R}_{\zeta}\right)$ denote the $\gamma$-bound of the set $\mathscr{R}_{\zeta}:=\{R(\lambda, A): \operatorname{Re} \lambda>\zeta\}$. By the resolvent identity and Proposition $3.1, t \mapsto R(i t, A) B$ belongs to $\gamma(\mathbb{R} ; H, E)$ and

$$
\begin{aligned}
\| R(i(\cdot), A) & B \|_{\gamma(\mathbb{R} ; H, E)} \\
& =\|[I-\omega R(i(\cdot), A)] R(\omega+i(\cdot), A) B\|_{\gamma(\mathbb{R} ; H, E)} \\
& \leqslant\left(1+|\omega| \gamma\left(\mathscr{R}_{\zeta}\right)\right)\|R(\omega+i(\cdot), A) B\|_{\gamma(\mathbb{R} ; H, E)} .
\end{aligned}
$$

Another application of Lemma 5.1 shows that $t \mapsto f_{B}(t):=S(t) B$ belongs to $\gamma\left(\mathbb{R}_{+} ; H, E\right)$.

Proof of Theorem 1.2. By Proposition 4.4 we have $S(\cdot) B \in \gamma\left(\mathbb{R}_{+} ; H, E\right)$. Hence $S(\cdot) B h \in \gamma\left(\mathbb{R}_{+} ; E\right)$ for all $h \in H$. Let $R_{B h}$ denote the operator in $\gamma\left(L^{2}\left(\mathbb{R}_{+}\right) ; E\right)$ represented by $S(\cdot) B h$. Theorem 1.2 is obtained by applying Theorem 3.4 to the operator $\Theta: H \rightarrow \gamma\left(L^{2}\left(\mathbb{R}_{+}\right) ; E\right), \Theta h:=R_{B h}$.

Proof of Theorem 1.3. By Proposition 4.4 we have $S(\cdot) x \in \gamma\left(\mathbb{R}_{+}, E\right)$ for all $x \in E$. Let $R_{x}$ denote the operator in $\gamma\left(L^{2}\left(\mathbb{R}_{+}\right) ; E\right)$ represented by $S(\cdot) x$. Theorem 1.3 is obtained by applying Theorem 3.4 to the operator $\Theta: E \rightarrow \gamma\left(L^{2}\left(\mathbb{R}_{+}\right) ; E\right)$, $\Theta x:=R_{x}$.

Remark 10. If $\left(\mathrm{SCP}_{B}\right)$ has a solution for all $\gamma$-radonifying operators $B \in \mathscr{L}(H, E)$, then for all $\delta>0$ the family $\{R(\lambda, A): \operatorname{Re} \lambda \geqslant \delta\}$ is $R$-bounded as a family of operators in $\mathscr{L}(\gamma(H, E))$ with $R$-bound of order $O(1 / \sqrt{\delta})$ as $\delta \downarrow 0$; here $R(\lambda, A) \in$ $\mathscr{L}(\gamma(H, E))$ is defined by the action $B \mapsto R(\lambda, A) B$. This is proved by extending Theorem 3.4 to the following more general situation. First, for an operator $B \in$ $\mathscr{L}\left(L^{2}\left(\mathbb{R}_{+} ; H\right), E\right)$ its the Laplace transform $\widehat{B}:\{\operatorname{Re} \lambda>0\} \rightarrow \mathscr{L}(H, E)$ is defined by

$$
\widehat{B}(\lambda) h:=B\left(e_{\lambda} \otimes h\right) .
$$

The Laplace transform $\widehat{\Theta}:\{\operatorname{Re} \lambda>0\} \rightarrow \mathscr{L}(F, \mathscr{L}(H, E))$ of a bounded operator $\Theta: F \rightarrow \mathscr{L}\left(L^{2}\left(\mathbb{R}_{+} ; H\right), E\right)$ is then defined by

$$
(\widehat{\Theta}(\lambda) y) h:=\widehat{\Theta y}(\lambda) h .
$$

If $\Theta$ takes values in $\gamma\left(L^{2}\left(\mathbb{R}_{+} ; H\right), E\right)$, then $\widehat{\Theta}$ takes values in $\mathscr{L}(F, \gamma(H, E))$. Theorem 3.4 extends to this situation mutatis mutandis.

Finally, Theorem 1.4 follows from Theorems 1.1 and 1.2, and Theorem 1.5 follows from Theorems 1.1, 1.3, and Corollary 4.6.

\section{REFERENCES}

[1] W. Arendt, Spectrum and growth of positive semigroups, in: "Evolution Equations" (Baton Rouge, 1992), Lecture Notes in Pure and Appl. Math., Vol. 168, Dekker, 1995, pp. 21-28.

[2] W. Arendt, C.J.K. Batty, M. Hieber, and F. Neubrander, "Vector-Valued Laplace Transforms and Cauchy Problems", Monographs in Mathematics, Vol. 96, Birkhäuser Verlag, 2001.

[3] V.I. Bogachev, "Gaussian Measures", Math. Surveys and Monographs, Vol. 62, Amer. Math. Soc., 1998.

[4] Z. Brzeźniak and J.M.A.M. van Neerven, Stochastic convolution in separable Banach spaces and the stochastic linear Cauchy problem, Studia Math. 143 (2000), 43-74. 
[5] Ph. Clément, B. de Pagter, F. Sukochev, H. Witvliet, Schauder decomposition and multiplier theorems, Studia Math. 138 (2000), 135-163.

[6] G. Da Prato and J. Zabczyk, "Stochastic Equations in Infinite Dimensions", Encyclopedia of Mathematics and its Applications, Cambridge University Press, 1992.

[7] G. Da Prato and J. Zabczyk, "Ergodicity for Infinite-Dimensional Systems", London Mathematical Society Lecture Note Series, Vol. 229, Cambridge University Press, 1996.

[8] R. Denk, M. Hieber, and J. Prüss, "R-Boundedness, Fourier Multipliers and Problems of Elliptic and Parabolic Type", Mem. Amer. Math. Soc., Vol. 166, 2003.

[9] J. Dettweiler, J.M.A.M. van Neerven, Continuity versus nonexistence for a class of linear stochastic Cauchy problems driven by a Brownian motion, to appear in Czech. Math. J.

[10] J. Dettweiler, J.M.A.M. van Neerven, and L. Weis, Space-time regularity of solutions of the parabolic stochastic Cauchy problem, in preparation.

[11] J. Diestel, H. Jarchow and A. Tonge, "Absolutely Summing Operators", Cambridge Studies in Adv. Math., Vol. 34, Cambridge University Press, 1995.

[12] L. Gearhart, Spectral theory for contraction semigroups on Hilbert spaces, Trans. Amer. Math. Soc. 236 (1978), 385-394.

[13] M. Girardi And L. Weis, Operator-valued Fourier multiplier theorems on $L_{p}(X)$ and geometry of Banach spaces, J. Funct. Anal. 204 (2003), 320-354.

[14] M. Girardi and L. Weis, Criteria for $R$-boundedness of operator families, in: "Evolution Equations, pp. 203-221, Lecture Notes in Pure and Appl. Math., Vol. 234, Dekker, 2003.

[15] B. Goldys And J.M.A.M. van Neerven, Transition semigroups of Banach space valued Ornstein-Uhlenbeck processes, Acta Applicandae Math. 76 (2003), 283-330.

[16] I.W. Herbst, The spectrum of Hilbert space semigroups, J. Operator Th. 10 (1983), 87-94.

[17] N.J. Kalton and L. Weis, Euclidean structures and applications to $H^{\infty}$-calculus, in preparation.

[18] N.J. Kalton and L. Weis, The $H^{\infty}$-functional calculus and square function estimates, in preparation.

[19] P.Ch. Kunstmann and L. Weis, "Maximal $L^{p}$-Regularity for Parabolic Equations, Fourier Multiplier Theorems and $H^{\infty}$-Functional Calculus", in: Springer Lect. Notes Math., Vol. 1855, Springer-Verlag, 2004, pp. 65-311.

[20] W. Linde And A. Pietsch, Mappings of Gaussian cylindrical measures in Banach spaces, Theory Probab. Appl. 19 (1974), 445-460.

[21] M. Ledoux and M. Talagrand, "Probability in Banach Spaces", Ergebnisse d. Math. u. ihre Grenzgebiete, Vol. 23, Springer-Verlag, 1991.

[22] J.M.A.M. van NeERven, "The Asymptotic Behaviour of Semigroups of Linear Operators", Operator Theory: Advances and Applications, Vol. 88, Birkhäuser Verlag, 1996.

[23] J.M.A.M. vAn NEERVEn, Uniqueness of invariant measures for the stochastic Cauchy problem in Banach spaces, in: L. Kérchy, C. Foiaş, I. Gohberg, H. Langer (eds.): "Recent Advances in Operator Theory and Related Topics: The Béla Szökefalvi-Nagy Memorial Volume", Operator Theory: Advances and Applications, Vol. 127, Birkhäuser Verlag, 2001, pp. 491-517.

[24] J.M.A.M. VAn NeERven AND L. Weis, Stochastic integration of functions with values in a Banach space, Studia Math. 166 (2005), 131-170.

[25] J.M.A.M. van NeERven And L. Weis, Weak limits and integrals of Gaussian covariances in Banach spaces, to appear in Probab. Math. Statist.

[26] J.M.A.M. van NEERVEN AND L. WeIs, On stochastic integration of operator-valued functions with respect to Banach space-valued Brownian motion, submitted for publication.

[27] G. PISIER, Some results on Banach spaces without local unconditional structure, Compositio Math. 37 (1978), 3-19.

[28] J. Prüss, On the spectrum of $C_{0}$-semigroups, Trans. Amer. Math. Soc. 284 (1984), 847-857.

[29] J. Rosiński And Z. Suchanecki, On the space of vector-valued functions integrable with respect to the white noise, Colloq. Math. XLIII (1980), 183-201.

[30] M. Slemrod, Asymptotic behavior of $C_{0}$ semi-groups as determined by the spectrum of the generator, Indiana Univ. Math. J. 25 (1976), 783-792.

[31] N.N. Vakhania, V.I. Tarieladze, and S.A. Chobanyan, "Probability Distributions in Banach Spaces", D. Reidel Publishing Company, Dordrecht-Boston-Lancaster-Tokyo, 1987.

[32] L. WEIS, Operator-valued Fourier multiplier theorems and maximal $L_{p}$-regularity, Math. Ann. 319 (2001), 735-758. 
[33] L. Weis and V. Wrobel, Asymptotic behavior of $C_{0}$-semigroups in Banach spaces, Proc. Amer. Math. Soc. 124 (1996), 3663-3671.

Delft Institute of Applied Mathematics, Technical University of Delft, P.O. Box 5031, 2600 GA Delft, The Netherlands

E-mail address: J.vanNeerven@math.tudelft.nl

Mathematisches Institut I, Technische Universität Karlsruhe, D-76128 KarlsRuhe, Germany

E-mail address: Lutz.Weis@math.uni-karlsruhe.de 